\title{
Inference for the Type-II Generalized Logistic Distribution with Progressive Hybrid Censoring
}

\author{
Mina Azizpour and Akbar Asgharzadeh* \\ University of Mazandaran
}

Received: 8/31/2016 Approved: 9/13/2017

\begin{abstract}
This article presents the analysis of the Type-II hybrid progressively censored data when the lifetime distributions of the items follow TypeII generalized logistic distribution. Maximum likelihood estimators (MLEs) are investigated for estimating the location and scale parameters. It is observed that the MLEs can not be obtained in explicit forms. We provide the approximate maximum likelihood estimators (AMLEs) by appropriately approximating the likelihood equations. Asymptotic confidence intervals based on MLEs and AMLEs and one bootstrap confidence interval are proposed. Estimation of the shape parameter is also discussed. Monte Carlo simulations are performed to compare the performances of the different methods and two real data sets have been analyzed for illustrative purposes.
\end{abstract}

Keywords. Maximum likelihood estimation; progressively Type-II hybrid censoring; Type-II generalized logistic distribution.

MSC 2010: 62N05, 62F10.

\footnotetext{
* Corresponding author

Copyright 1 2019, ASP Ins. This open-access article is published under the terms of the Creative Commons AttributionNonCommercial 4.0 International License which permits Share (copy and redistribute the material in any medium or format) and Adapt (remix, transform, and build upon the material) under the Attribution-NonCommercial terms.
} 


\section{Introduction}

The Type-II generalized logistic distribution (GLD) has the probability density function (pdf)

$$
g(y ; \mu, \sigma)=\frac{b}{\sigma} \cdot \frac{e^{-b\left(\frac{y-\mu}{\sigma}\right)}}{\left(1+e^{-\frac{y-\mu}{\sigma}}\right)^{b+1}}, \quad-\infty<y<\infty ; b>0,
$$

and the cumulative distribution function (cdf)

$$
G(y ; \mu, \sigma)=1-\left(\frac{e^{-\frac{y-\mu}{\sigma}}}{1+e^{-\frac{y-\mu}{\sigma}}}\right)^{b}, \quad-\infty<y<\infty ; b>0 .
$$

Balakrishnan and Leung (1988) derived the above distribution as one of three generalized forms of the standard logistic distribution. This distribution is obtained by compounding an extreme value distribution of the double exponential type with a gamma distribution. The Type-II GLD is negatively skewed when $0<b<1$ and positively skewed when $b>1$, and for $b=1$, it becomes the standard logistic distribution. This distribution is unimodal and log-concave and it can be used to model both left an right skewed data (see Balakrishnan and Hossain, 2007).

Censoring is very common in life-testing and reliability experiments to save total time on the test, to save experimental units for future use, and to save on the cost of the experiment. The two most common censoring schemes are Type-I and Type-II censoring. Consider a sample of $n$ units placed on a life-test at time 0 . In Type-I censoring scheme, a time $T$, independent of the failure times, is pre-fixed so that beyond this time no failures will be observed, that is, the life-testing experiment terminates at time $T$. In Type-II censoring scheme, the number of observed failures is fixed, say $r(r \leqslant n)$, and the life-testing experiment stops when the $r$ th failure takes place. The mixture of Type-I and Type-II censoring schemes is known as the hybrid censoring scheme which was first introduced by Epstein (1954). Many articles have considered this censoring scheme. See, for example, Chen and Bhattacharya (1988), Draper and Guttman (1987), Fairbanks et al. (1982), Gupta and Kundu (1998), Kundu (2007), Asgharzadeh et al. (2015), Asgharzadeh et al. (2017) and Valiollahi et al. (2017).

Conventional Type-I, Type-II censoring and hybrid censoring schemes do 
not have the flexibility of allowing the removal of experimental units before the termination of the experiment. Progressive Type-II censoring is a more general censoring scheme which has this flexibility. It can be described as follows. Consider an experiment in which $n(n>m)$ units are put on a life test at time 0 . Immediately following the first failure, $R_{1}$ units from the remaining $n-1$ surviving units are randomly selected and removed from the test. Then, immediately following the second failure, $R_{2}$ units from the remaining $n-2-R_{1}$ surviving units are randomly selected and removed from the test. This process continues until, at the time of the $m$ th failure, all the remaining $R_{m}=n-m-R_{1}-R_{2}-\cdots-R_{m-1}$ units are removed from the test. The $R_{i}$ 's are fixed prior to the study. For more details, the readers may refer to the Balakrishnan and Aggarwala (2000).

As mentioned by Kundu and Joarder (2006), the major drawback of the Type-II progressive censoring is that it can take a lot of time to get to the $m$ th failure time. For this reason, they proposed the Type-II progressively hybrid censoring scheme in which the life-testing experiment is terminated after a random time $\min \left\{Y_{m: m: n}, T\right\}$ where the time point $T$ and the integers of $n$ and $m, 1 \leqslant m \leqslant n$ are fixed prior to the experiment, and $Y_{1: m: n} \leqslant Y_{2: m: n} \leqslant$ $\ldots \leqslant Y_{m: m: n}$ are the ordered failure times resulting from the experiment. It can be described as follows: consider $n$ identical items which put on a life test. The integers $R_{1}, \ldots, R_{m}$ satisfying $R_{1}+\cdots+R_{m}+m=n$ are fixed at the beginning of the experiment. At the time of first failure, say $Y_{1: m: n}, R_{1}$ of the remaining units are randomly selected and removed. Similarly, at the time of the second failure, say $Y_{2: m: n}, R_{2}$ of the remaining units are randomly selected and removed and so on. If the $m$ th failure $Y_{m: m: n}$, occurs before the time point $T$ (i.e., $Y_{m: m: n}<T$ ), the experiment stops at the time point $Y_{m: m: n}$. On the other hand, if the $m$ th failure does not occur before time point $T$ and only $J$ failures occur before the time point $T$, then at the time point $T$, all the remaining $R_{J}^{*}=n-R_{1}-\cdots-R_{J}-J$ units are removed and the experiment terminates at the time point $T$. For more details and some recent references on progressive hybrid censoring schemes, see Kundu and Joarder (2006), Lin et al. (2009), Joarder et al. (2009), Bayat Mokhtari et al. (2011), Hemmati and Khorram (2013), Gurunlu Alma and Arabi Belaghi. (2016) and Kayal et al. (2017).

We denote two cases mentioned above as Case I and Case II, respectively. Therefore, in the presence of progressively Type-II hybrid censoring scheme, we have one of the following types of observations: 
Case I: $\left\{Y_{1: m: n}, \ldots, Y_{m: m: n}\right\} \quad$ if $Y_{m: m: n}<T$,

Case II: $\left\{Y_{1: m: n}, \ldots, Y_{J: m: n}\right\} \quad$ if $Y_{J: m: n}<T<Y_{J+1: m: n}$. Note that for Case II

$$
Y_{J: m: n}<T<Y_{J+1: m: n}<\cdots<Y_{m: m: n}
$$

and $Y_{J+1: m: n}, \ldots, Y_{m: m: n}$ are not observed.

In this article, we consider progressively Type-II hybrid censored data from the Type-II GLD. In Section 2, We provide the MLEs of the location and scale parameters. It is observed that the MLEs can not be obtained in explicit forms. Section 3 provides explicit estimators by appropriately approximating the likelihood equations. Asymptotic confidence intervals based on MLEs and AMLEs and one bootstrap confidence interval are provided in Section 4. In Section 5, we provide the results of a simulation study in order to evaluate the performance of the approximate estimators and the MLEs determined by numerical methods. In Section 6, we present two numerical examples to illustrate the methods of inference discussed in the preceding sections. Estimation of the shape parameter is also discussed in this section. Finally, the conclusions are presented in Section 7.

\section{Maximum Likelihood Estimators}

If $Y$ has the Type-II GLD with the location parameter $\mu$ and the scale parameter $\sigma$, then the random variable $X=\frac{Y-\mu}{\sigma}$ has the standard Type-II GLD with pdf and cdf given by

$$
f(x)=\frac{b e^{-b x}}{\left(1+e^{-x}\right)^{b+1}}, \quad-\infty<x<\infty ; b>0,
$$

and

$$
F(x)=1-\left(\frac{e^{-x}}{1+e^{-x}}\right)^{b}, \quad-\infty<x<\infty ; b>0,
$$

respectively.

Based on the observed progressively Type-II hybrid censored data from (1), the likelihood function for Case I is given by 


$$
L(\mu, \sigma)=k_{1} \prod_{i=1}^{m} g\left(y_{i: m: n}\right)\left[1-G\left(y_{i: m: n}\right)\right]^{R_{i}}
$$

where $k_{1}=\prod_{i=1}^{m}\left[n-\sum_{k=1}^{i-1}\left(1+R_{k}\right)\right]$. While for Case II, it is

$$
L(\mu, \sigma)=k_{2} \prod_{i=1}^{J} g\left(y_{i: m: n}\right)\left[1-G\left(y_{i: m: n}\right)\right]^{R_{i}}[1-G(T)]^{R_{J}^{*}}
$$

if $J>0$, where $k_{2}=\prod_{i=1}^{J}\left[n-\sum_{k=1}^{i-1}\left(1+R_{k}\right)\right]$. Note that when $J=0$, the MLEs do not exist. So from now on, it is assumed that $J>0$.

Now the likelihood function may be rewritten for Case I as

$$
L(\mu, \sigma)=k_{1} \sigma^{-m} \prod_{i=1}^{m} f\left(x_{i: m: n}\right)\left[1-F\left(x_{i: m: n}\right)\right]^{R_{i}},
$$

while for Case II, it may be rewritten as

$$
L(\mu, \sigma)=k_{2} \sigma^{-J} \prod_{i=1}^{J} f\left(x_{i: m: n}\right)\left[1-F\left(x_{i: m: n}\right)\right]^{R_{i}}[1-F(S)]^{R_{J}^{*}},
$$

where $x_{i: m: n}=\frac{y_{i: m: n}-\mu}{\sigma}$ and $S=\frac{T-\mu}{\sigma}$. To simplify the notation, we will use $x_{i}$ and $y_{i}$ in place of $x_{i: m: n}$ and $y_{i: m: n}$, respectively.

The log-likelihood function for Case I is

$$
\ln L(\mu, \sigma)=\ln \left(k_{1}\right)-m \ln \sigma+\sum_{i=1}^{m} \ln f\left(x_{i}\right)+\sum_{i=1}^{m} R_{i} \ln \left[1-F\left(x_{i}\right)\right],
$$

and for Case II, it is

$\ln L(\mu, \sigma)=\ln \left(k_{2}\right)-J \ln \sigma+\sum_{i=1}^{J} \ln f\left(x_{i}\right)+\sum_{i=1}^{J} R_{i} \ln \left[1-F\left(x_{i}\right)\right]+R_{J}^{*} \ln (1-F(S))$.

From (5) and (6), we obtain the likelihood equations for Case I as

$$
\frac{\partial \ln L(\mu, \sigma)}{\partial \mu}=-\frac{1}{\sigma} \sum_{i=1}^{m} \frac{f^{\prime}\left(x_{i}\right)}{f\left(x_{i}\right)}+\frac{1}{\sigma} \sum_{i=1}^{m} R_{i} \frac{f\left(x_{i}\right)}{1-F\left(x_{i}\right)}=0,
$$




$$
\frac{\partial \ln L(\mu, \sigma)}{\partial \sigma}=-\frac{m}{\sigma}-\frac{1}{\sigma} \sum_{i=1}^{m} x_{i} \frac{f^{\prime}\left(x_{i}\right)}{f\left(x_{i}\right)}+\frac{1}{\sigma} \sum_{i=1}^{m} R_{i} x_{i} \frac{f\left(x_{i}\right)}{1-F\left(x_{i}\right)}=0,
$$

and for Case II as

$$
\begin{aligned}
\frac{\partial \ln L(\mu, \sigma)}{\partial \mu}=-\frac{1}{\sigma} \sum_{i=1}^{J} & \frac{f^{\prime}\left(x_{i}\right)}{f\left(x_{i}\right)}+\frac{1}{\sigma} \sum_{i=1}^{J} R_{i} \frac{f\left(x_{i}\right)}{1-F\left(x_{i}\right)}+\frac{1}{\sigma} R_{J}^{*} \frac{f(S)}{1-F(S)}=0 \\
\frac{\partial \ln L(\mu, \sigma)}{\partial \sigma}= & -\frac{J}{\sigma}-\frac{1}{\sigma} \sum_{i=1}^{J} x_{i} \frac{f^{\prime}\left(x_{i}\right)}{f\left(x_{i}\right)}+\frac{1}{\sigma} \sum_{i=1}^{J} R_{i} x_{i} \frac{f\left(x_{i}\right)}{1-F\left(x_{i}\right)} \\
& +\frac{1}{\sigma} R_{J}^{*} S \frac{f(S)}{1-F(S)}=0 .
\end{aligned}
$$

Equations (7)-(10) do not appear to admit explicit solutions for $\mu$ and $\sigma$ and hence must be solved numerically to obtain the MLEs of the parameters. We have to employ some iterative methods, such as Newton- Raphson method, to obtain the MLEs of $\mu$ and $\sigma$ which requires a starting value near the global maximum.

\section{Approximate Estimators}

The likelihood equations in (7)-(10) are nonlinear and do not admit explicit solutions because of the terms of $\frac{f^{\prime}\left(x_{i}\right)}{f\left(x_{i}\right)}\left(\right.$ say $\left.h_{1}\left(x_{i}\right)\right), \frac{f\left(x_{i}\right)}{1-F\left(x_{i}\right)}$ (say $h_{2}\left(x_{i}\right)$ ) and $\frac{f(S)}{1-F(S)}\left(\right.$ say $\left.h_{2}(S)\right)$. We approximate the functions $h_{1}\left(x_{i}\right)$ and $h_{2}\left(x_{i}\right)$ by expanding them in a Taylor series around the point $\nu_{i: m: n}$, where

$$
\nu_{i: m: n}=E\left(X_{i: m: n}\right) \approx F^{-1}\left(\alpha_{i: m: n}\right),
$$

and

$$
\alpha_{i: m: n}=1-\prod_{j=m-i+1}^{m} \frac{j+R_{m-j+1}+\cdots+R_{m}}{j+1+R_{m-j+1}+\cdots+R_{m}}, \quad i=1, \ldots, m,
$$

(see Balakrishnan and Aggarwala (2000) for reasoning). Here we also approximate $h_{2}(S)$ by expanding it in a Taylor series around the point $\xi_{J: m: n}$, where 


$$
\xi_{J: m: n} \approx F^{-1}\left(\frac{\alpha_{J: m: n}+\alpha_{J+1: m: n}}{2}\right) .
$$

For the Type-II GLD, we have

$$
F^{-1}(u)=\ln \left(\frac{1-(1-u)^{1 / b}}{(1-u)^{1 / b}}\right) .
$$

Now, by expanding the functions $h_{1}\left(x_{i}\right)$ and $h_{2}\left(x_{i}\right)$ around the point $\nu_{i: m: n}$ and $h_{2}(S)$ around the point $\xi_{J: m: n}$ and keeping only two terms we may then approximate these functions by

$$
\begin{aligned}
h_{1}\left(x_{i}\right) & \approx h_{1}\left(\nu_{i: m: n}\right)+h_{1}^{\prime}\left(\nu_{i: m: n}\right)\left(x_{i}-\nu_{i: m: n}\right), \\
& =\alpha_{i}-\beta_{i} x_{i}, \\
h_{2}\left(x_{i}\right) & \approx h_{2}\left(\nu_{i: m: n}\right)+h_{2}^{\prime}\left(\nu_{i: m: n}\right)\left(x_{i}-\nu_{i: m: n}\right), \\
& =\delta_{i}+\gamma_{i} x_{i},
\end{aligned}
$$

and

$$
\begin{aligned}
h_{2}(S) & \approx h_{2}\left(\xi_{J: m: n}\right)+h_{2}^{\prime}\left(\xi_{J: m: n}\right)\left(S-\xi_{J: m: n}\right), \\
& =\delta_{d}^{*}+\gamma_{d}^{*} S,
\end{aligned}
$$

where

$$
\begin{aligned}
\alpha_{i} & =h_{1}\left(\nu_{i: m: n}\right)-\nu_{i: m: n} h_{1}^{\prime}\left(\nu_{i: m: n}\right), \\
\beta_{i} & =-h_{1}^{\prime}\left(\nu_{i: m: n}\right), \\
\delta_{i} & =h_{2}\left(\nu_{i: m: n}\right)-\nu_{i: m: n} h_{2}^{\prime}\left(\nu_{i: m: n}\right), \\
\delta_{d}^{*} & =h_{2}\left(\xi_{J: m: n}\right)-\xi_{J: m: n} h_{2}^{\prime}\left(\xi_{J: m: n}\right), \\
\gamma_{d}^{*} & =h_{2}^{\prime}\left(\xi_{J: m: n}\right) .
\end{aligned}
$$

It is easy to show that $\beta_{i}>0, \gamma_{i}>0$ and $\gamma_{d}^{*}>0$. The proofs are provided in the Appendix.

Using (11), (12) and (13), we approximate the likelihood equations (7) 
and (8) for Case I by

$$
\begin{gathered}
\frac{\partial \ln L}{\partial \mu} \approx-\frac{1}{\sigma}\left[\sum_{i=1}^{m}\left(\alpha_{i}-\beta_{i} x_{i}\right)-\sum_{i=1}^{m} R_{i}\left(\delta_{i}+\gamma_{i} x_{i}\right)\right]=0, \\
\frac{\partial \ln L}{\partial \sigma} \approx-\frac{1}{\sigma}\left[m+\sum_{i=1}^{m} x_{i}\left(\alpha_{i}-\beta_{i} x_{i}\right)-\sum_{i=1}^{m} R_{i} x_{i}\left(\delta_{i}+\gamma_{i} x_{i}\right)\right]=0,
\end{gathered}
$$

and the likelihood equations (9) and (10) for Case II by

$$
\begin{gathered}
\frac{\partial \ln L}{\partial \mu} \approx-\frac{1}{\sigma}\left[\sum_{i=1}^{J}\left(\alpha_{i}-\beta_{i} x_{i}\right)-\sum_{i=1}^{J} R_{i}\left(\delta_{i}+\gamma_{i} x_{i}\right)-R_{J}^{*}\left(\delta_{d}^{*}+\gamma_{d}^{*} S\right)\right]=0 \\
\frac{\partial \ln L}{\partial \sigma} \approx-\frac{1}{\sigma}\left[J+\sum_{i=1}^{J} x_{i}\left(\alpha_{i}-\beta_{i} x_{i}\right)-\sum_{i=1}^{J} R_{i} x_{i}\left(\delta_{i}+\gamma_{i} x_{i}\right)\right. \\
\left.-R_{J}^{*} S\left(\delta_{d}^{*}+\gamma_{d}^{*} S\right)\right]=0
\end{gathered}
$$

Equations (14) and (16) may now be rewritten as

$$
\begin{gathered}
\sum_{i=1}^{m}\left(\alpha_{i}-R_{i} \delta_{i}\right)-\sum_{i=1}^{m}\left(\beta_{i}+R_{i} \gamma_{i}\right)\left(\frac{y_{i}-\mu}{\sigma}\right)=0, \\
\sum_{i=1}^{J}\left(\alpha_{i}-R_{i} \delta_{i}\right)-\sum_{i=1}^{J}\left(\beta_{i}+R_{i} \gamma_{i}\right)\left(\frac{y_{i}-\mu}{\sigma}\right)-R_{J}^{*} \delta_{d}^{*}-R_{J}^{*} \gamma_{d}^{*}\left(\frac{T-\mu}{\sigma}\right)=0,
\end{gathered}
$$

which yields the estimator of $\mu$ for Case I as

$$
\widetilde{\mu_{I}}=K_{I}-L_{I} \sigma,
$$

where

$$
K_{I}=\frac{\sum_{i=1}^{m}\left(\beta_{i}+R_{i} \gamma_{i}\right) y_{i}}{\sum_{i=1}^{m}\left(\beta_{i}+R_{i} \gamma_{i}\right)},
$$




$$
L_{I}=\frac{\sum_{i=1}^{m}\left(\alpha_{i}-R_{i} \delta_{i}\right)}{\sum_{i=1}^{m}\left(\beta_{i}+R_{i} \gamma_{i}\right)}
$$

and for Case II as

$$
\widetilde{\mu_{I I}}=K_{I I}-L_{I I} \sigma
$$

where

$$
\begin{array}{r}
K_{I I}=\frac{\sum_{i=1}^{J}\left(\beta_{i}+R_{i} \gamma_{i}\right) y_{i}+R_{J}^{*} \gamma_{d}^{*} T}{\sum_{i=1}^{J}\left(\beta_{i}+R_{i} \gamma_{i}\right)+R_{J}^{*} \gamma_{d}^{*}}, \\
L_{I I}=\frac{\sum_{i=1}^{J}\left(\alpha_{i}-R_{i} \delta_{i}\right)-R_{J}^{*} \delta_{d}^{*}}{\sum_{i=1}^{J}\left(\beta_{i}+R_{i} \gamma_{i}\right)+R_{J}^{*} \gamma_{d}^{*}} .
\end{array}
$$

Equation (15) may be written as

$$
m+\sum_{i=1}^{m}\left(\alpha_{i}-R_{i} \delta_{i}\right)\left(\frac{y_{i}-\mu}{\sigma}\right)-\sum_{i=1}^{m}\left(\beta_{i}+R_{i} \gamma_{i}\right)\left(\frac{y_{i}-\mu}{\sigma}\right)^{2}=0 .
$$

Replacing $\mu$ by $K_{I}-L_{I} \sigma$, we derive the AMLE $\sigma$ for Case I as

$$
\widetilde{\sigma_{I}}=\frac{-A_{I 1}+\sqrt{A_{I 1}^{2}+4 m A_{I 2}}}{2 m},
$$

where

$$
\begin{aligned}
& A_{I 1}=\sum_{i=1}^{m}\left(\alpha_{i}-R_{i} \delta_{i}\right)\left(y_{i}-K_{I}\right) \\
& A_{I 2}=\sum_{i=1}^{m}\left(\beta_{i}+R_{i} \gamma_{i}\right)\left(y_{i}-K_{I}\right)^{2} .
\end{aligned}
$$

Similarly, the equation (17) may be written as

$$
\begin{aligned}
J+\sum_{i=1}^{J}\left(\alpha_{i}-R_{i} \delta_{i}\right)\left(\frac{y_{i}-\mu}{\sigma}\right) & -\sum_{i=1}^{J}\left(\beta_{i}+R_{i} \gamma_{i}\right)\left(\frac{y_{i}-\mu}{\sigma}\right)^{2} \\
& -R_{J}^{*} \delta_{d}^{*}\left(\frac{T-\mu}{\sigma}\right)-R_{J}^{*} \gamma_{d}^{*}\left(\frac{T-\mu}{\sigma}\right)^{2}=0 .
\end{aligned}
$$

Replacing $\mu$ by $K_{I I}-L_{I I} \sigma$, we derive the AMLE $\sigma$ for Case II as

$$
\widetilde{\sigma_{I I}}=\frac{-A_{I I 1}+\sqrt{A_{I I 1}^{2}+4 J A_{I I 2}}}{2 J},
$$


where

$$
\begin{gathered}
A_{I I 1}=\sum_{i=1}^{J}\left(\alpha_{i}-R_{i} \delta_{i}\right)\left(y_{i}-K_{I I}\right)-R_{J}^{*} \delta_{d}^{*}\left(T-K_{I I}\right) \\
A_{I I 2}=\sum_{i=1}^{J}\left(\beta_{i}+R_{i} \gamma_{i}\right)\left(y_{i}-K_{I I}\right)^{2}+R_{J}^{*} \gamma_{d}^{*}\left(T-K_{I I}\right)^{2} .
\end{gathered}
$$

It should be mentioned here that upon solving equation (20) for $\sigma$ we obtain a quadratic equation in $\sigma$ which has two roots; however, one of them drops out, since $\beta_{i}>0, \gamma_{i}>0$ and $\gamma_{d}^{*}>0$, and hence $A_{I 2}>0$. Similarly, since $A_{I I 2}>0$, only one root in (22) is admissible. The approximated MLEs in (18), (19), (21), (23) can be used as the initiate values in the iterative procedure for solving the likelihood equations (7)-(10).

Note that the approximate MLEs depend on the shape parameter $b$. When $b=1$, the results for the standard logistic distribution are obtained as a special case. When the parameter $b$ is unknown, we may use the profile likelihood function to obtain its estimate. For fixed $b$, the approximate MLEs $\widetilde{\mu}=\widetilde{\mu}(b)$ and $\widetilde{\sigma}=\widetilde{\sigma}(b)$ are given as described above. Thus, the maximum profile likelihood estimate $\widetilde{b}_{p}$ may be obtained by maximizing the likelihood $L(\widetilde{\mu}(b), \widetilde{\sigma}(b), b)$ with respect to $b$. In Section 6 , the profile likelihood method and some other methods have been proposed to estimate the shape parameter $b$.

\section{Confidence Intervals}

In this section, the aim is that to provide the confidence intervals for $\mu$ and $\sigma$ based on the progressively Type-II hybrid censored samples. Since the exact confidence intervals for $\mu$ and $\sigma$ are difficult to obtain, we propose asymptotic confidence intervals based on the asymptotic distributions of MLE and AMLE. Further, one bootstrap confidence interval is also proposed.

\subsection{Asymptotic Confidence Intervals}

Here, we compute the observed Fisher information based on the likelihood as well as the approximate likelihood equations. These will enable us to develop pivotal quantities based on the limiting normal distribution and then obtain the asymptotic confidence intervals based on MLEs and AMLEs. Balakrishnan and Kannan (2000), Asgharzadeh (2006) and Balakrishnan 
and Hossain (2007) have discussed the asymptotic confidence intervals based on MLEs and AMLEs for logistic and generalized logistic distributions based on progressively Type-II censored samples.

From the log-likelihood functions in (7) and (8) we obtain the observed Fisher information for Case I as

$$
\begin{aligned}
& \frac{\partial^{2} \ln L}{\partial \mu^{2}}=\frac{1}{\sigma^{2}} \sum_{i=1}^{m} h_{1}^{\prime}\left(x_{i}\right)-\frac{1}{\sigma^{2}} \sum_{i=1}^{m} R_{i} h_{2}^{\prime}\left(x_{i}\right), \\
& \frac{\partial^{2} \ln L}{\partial \mu \partial \sigma}=\frac{1}{\sigma^{2}} \sum_{i=1}^{m} h_{1}\left(x_{i}\right)-\frac{1}{\sigma^{2}} \sum_{i=1}^{m} R_{i} h_{2}\left(x_{i}\right)+\frac{1}{\sigma^{2}} \sum_{i=1}^{m} x_{i} h_{1}^{\prime}\left(x_{i}\right)-\frac{1}{\sigma^{2}} \sum_{i=1}^{m} R_{i} x_{i} h_{2}^{\prime}\left(x_{i}\right) \\
& \frac{\partial^{2} \ln L}{\partial \sigma^{2}}=\frac{m}{\sigma^{2}}+\frac{2}{\sigma^{2}} \sum_{i=1}^{m} x_{i} h_{1}\left(x_{i}\right)+\frac{1}{\sigma^{2}} \sum_{i=1}^{m} x_{i}^{2} h_{1}^{\prime}\left(x_{i}\right)-\frac{2}{\sigma^{2}} \sum_{i=1}^{m} R_{i} x_{i} h_{2}\left(x_{i}\right) \\
& -\frac{1}{\sigma^{2}} \sum_{i=1}^{m} R_{i} x_{i}^{2} h_{2}^{\prime}\left(x_{i}\right) \text {. }
\end{aligned}
$$

From the log-likelihood functions in (9) and (10), we obtain the observed Fisher information for Case II as

$$
\begin{gathered}
\frac{\partial^{2} \ln L}{\partial \mu^{2}}=\frac{1}{\sigma^{2}} \sum_{i=1}^{J} h_{1}^{\prime}\left(x_{i}\right)-\frac{1}{\sigma^{2}} \sum_{i=1}^{J} R_{i} h_{2}^{\prime}\left(x_{i}\right)-\frac{1}{\sigma^{2}} R_{J}^{*} h_{2}^{\prime}(S), \\
\frac{\partial^{2} \ln L}{\partial \mu \partial \sigma}=\frac{1}{\sigma^{2}} \sum_{i=1}^{J} h_{1}\left(x_{i}\right)-\frac{1}{\sigma^{2}} \sum_{i=1}^{J} R_{i} h_{2}\left(x_{i}\right)+\frac{1}{\sigma^{2}} \sum_{i=1}^{m} x_{i} h_{1}^{\prime}\left(x_{i}\right) \\
-\frac{1}{\sigma^{2}} \sum_{i=1}^{J} R_{i} x_{i} h_{2}^{\prime}\left(x_{i}\right)-\frac{1}{\sigma^{2}} R_{J}^{*} h_{2}(S)-\frac{1}{\sigma^{2}} R_{J}^{*} S h_{2}^{\prime}(S), \\
\frac{\partial^{2} \ln L}{\partial \sigma^{2}}=\frac{J}{\sigma^{2}}+\frac{2}{\sigma^{2}} \sum_{i=1}^{J} x_{i} h_{1}\left(x_{i}\right)+\frac{1}{\sigma^{2}} \sum_{i=1}^{J} x_{i}^{2} h_{1}^{\prime}\left(x_{i}\right)-\frac{2}{\sigma^{2}} \sum_{i=1}^{J} R_{i} x_{i} h_{2}\left(x_{i}\right) \\
-\frac{1}{\sigma^{2}} \sum_{i=1}^{m} R_{i} x_{i}^{2} h_{2}^{\prime}\left(x_{i}\right)-\frac{2}{\sigma^{2}} R_{J}^{*} S h_{2}(S)-\frac{1}{\sigma^{2}} R_{J}^{*} S^{2} h_{2}^{\prime}(S) .
\end{gathered}
$$


We may derive similar expressions for the observed fisher information based on the approximate likelihood equations. Using the approximate likelihood equations (14) and (15), we derive the observed fisher information for Case I as:

$$
\begin{gathered}
\frac{\partial^{2} \ln L}{\partial \mu^{2}} \approx-\frac{1}{\sigma^{2}} \sum_{i=1}^{m}\left(\beta_{i}+R_{i} \gamma_{i}\right), \\
\frac{\partial^{2} \ln L}{\partial \mu \partial \sigma} \approx \frac{1}{\sigma^{2}} \sum_{i=1}^{m}\left(\alpha_{i}-R_{i} \delta_{i}\right)-\frac{2}{\sigma^{2}} \sum_{i=1}^{m}\left(\beta_{i}+R_{i} \gamma_{i}\right) x_{i}, \\
\frac{\partial^{2} \ln L}{\partial \sigma^{2}} \approx \frac{m}{\sigma^{2}}+\frac{2}{\sigma^{2}} \sum_{i=1}^{m}\left(\alpha_{i}-R_{i} \delta_{i}\right) x_{i}-\frac{3}{\sigma^{2}} \sum_{i=1}^{m}\left(\beta_{i}+R_{i} \gamma_{i}\right) x_{i}^{2} .
\end{gathered}
$$

and for Case II (using (16) and (17)) as

$$
\begin{gathered}
\frac{\partial^{2} \ln L}{\partial \mu^{2}} \approx-\frac{1}{\sigma^{2}} \sum_{i=1}^{J}\left(\beta_{i}+R_{i} \gamma_{i}\right)-\frac{1}{\sigma^{2}} R_{J}^{*} \gamma_{d}^{*}, \\
\frac{\partial^{2} \ln L}{\partial \mu \partial \sigma} \approx \frac{1}{\sigma^{2}} \sum_{i=1}^{J}\left(\alpha_{i}-R_{i} \delta_{i}\right)-\frac{2}{\sigma^{2}} \sum_{i=1}^{J}\left(\beta_{i}+R_{i} \gamma_{i}\right) x_{i}-\frac{1}{\sigma^{2}} R_{J}^{*} \delta_{d}^{*}-\frac{2}{\sigma^{2}} R_{J}^{*} \gamma_{d}^{*} S, \\
\frac{\partial^{2} \ln L}{\partial \sigma^{2}} \approx \frac{J}{\sigma^{2}}+\frac{2}{\sigma^{2}} \sum_{i=1}^{J}\left(\alpha_{i}-R_{i} \delta_{i}\right) x_{i}-\frac{3}{\sigma^{2}} \sum_{i=1}^{J}\left(\beta_{i}+R_{i} \gamma_{i}\right) x_{i}^{2}-\frac{2}{\sigma^{2}} R_{J}^{*} \delta_{d}^{*}-\frac{3}{\sigma^{2}} R_{J}^{*} \gamma_{d}^{*} S^{2} .
\end{gathered}
$$

If we denote $-\frac{\partial^{2} \ln L}{\partial \mu^{2}}=\frac{V_{1}}{\sigma^{2}}, \quad-\frac{\partial^{2} \ln L}{\partial \mu \partial \sigma}=\frac{V_{2}}{\sigma^{2}}$, and $-\frac{\partial^{2} \ln L}{\partial \sigma^{2}}=\frac{V_{3}}{\sigma^{2}}$, then the observed information matrix can be inverted to obtain the asymptotic variance covariance matrix of the estimators as

$$
\left\{\frac{1}{\sigma^{2}}\left(\begin{array}{ll}
V_{1} & V_{2} \\
V_{2} & V_{3}
\end{array}\right)\right\}^{-1}=\sigma^{2}\left(\begin{array}{ll}
V^{11} & V^{12} \\
V^{12} & V^{22}
\end{array}\right)
$$

where

$$
V^{11}=\frac{V_{3}}{V_{1} V_{3}-V_{2}^{2}}, \quad V^{12}=-\frac{V_{2}}{V_{1} V_{3}-V_{2}^{2}}, \quad V^{22}=\frac{V_{1}}{V_{1} V_{3}-V_{2}^{2}} .
$$

Similarly $V_{*}^{11}, V_{*}^{12}, V_{*}^{22}$ can be obtained from the observed Fisher information for the approximate likelihood equations.

Let us now obtain the asymptotic confidence intervals based on the MLEs. 
Under some regularity conditions (e.g., see Ferguson (1996), p. 121), since the $\operatorname{MLE}\left(\begin{array}{c}\hat{\mu} \\ \hat{\sigma}\end{array}\right)$ is asymptotically normally distributed, we have the asymptotic distribution of

$$
P_{1}=\frac{\hat{\mu}-\mu}{\hat{\sigma} \sqrt{V^{11}}}, \quad P_{2}=\frac{\hat{\sigma}-\sigma}{\hat{\sigma} \sqrt{V^{22}}}
$$

to be standard normal. Using the pivotal quantities $P_{1}$ and $P_{2}$, the 100(1$\alpha) \%$ asymptotic confidence intervals for $\mu$ and $\sigma$ based on the MLEs are

$$
\left(\widehat{\mu}-z_{\frac{\alpha}{2}} \widehat{\sigma} \sqrt{V^{11}}, \widehat{\mu}+z_{\frac{\alpha}{2}} \widehat{\sigma} \sqrt{V^{11}}\right)
$$

and

$$
\left(\widehat{\sigma}-z_{\frac{\alpha}{2}} \widehat{\sigma} \sqrt{V^{22}}, \widehat{\sigma}+z_{\frac{\alpha}{2}} \widehat{\sigma} \sqrt{V^{22}}\right),
$$

respectively. Similarly, the asymptotic confidence intervals based on the AMLEs $\widetilde{\mu}$ and $\widetilde{\sigma}$ can be obtained.

\subsection{Bootstrap Confidence Interval}

The bootstrap re-sampling method was proposed by Efron (1979). It is commonly used to estimate confidence intervals, but it can be used also to estimate the bias and variance of an estimator. The method treats the observed sample as if it represented the population. From the information obtained from such a sample, bootstrap samples of similar size to that of the observed sample are generated, from which it is possible to estimate various characteristics of the population, such as mean, variance, percentiles and so on. One of the bootstrap methods that are widely used in practice is the percentile bootstrap (Boot-p) proposed by Efron (1982). We use the following steps for obtaining the Boot-p confidence intervals for $\mu$ and $\sigma$ :

- Estimate $\mu$ and $\sigma$, say $\widetilde{\mu}$ and $\widetilde{\sigma}$, from the sample using (18), (19), (21) and (23).

- Generate a bootstrap sample $\left\{Y_{1: m: n}^{*}, \ldots, Y_{k: m: n}^{*}\right\}$ by using $\widetilde{\mu}$ and $\widetilde{\sigma}$, $R_{1}, \ldots, R_{m}$ and $T$, where $k=m$ for Case I, and $K=J$ for Case II. Obtain the bootstrap estimate of $\mu$ and $\sigma$, say $\widetilde{\mu}^{*}$ and $\widetilde{\sigma}^{*}$ using the bootstrap sample. 
- Repeat the above step NBOOT times.

- Order $\widetilde{\mu}_{1}^{*}, \ldots, \widetilde{\mu}_{N B O O T}^{*}$ and $\widetilde{\sigma}_{1}^{*}, \ldots, \widetilde{\sigma}_{N B O O T}^{*}$ as $\widetilde{\mu}_{(1)}^{*}, \ldots, \widetilde{\mu}_{(N B O O T)}^{*}$ and $\tilde{\sigma}_{(1)}^{*}, \ldots, \widetilde{\sigma}_{(N B O O T)}^{*}$.

Then, the 100(1- $\alpha) \%$ bootstrap confidence intervals for $\mu$ and $\sigma$ become

$$
\left(\widetilde{\mu^{*}}{ }_{\text {Boot }-p}\left(\frac{\alpha}{2}\right),{\widetilde{\mu^{*}}}_{\text {Boot }-p}\left(1-\frac{\alpha}{2}\right)\right)
$$

and

$$
\left(\widetilde{\sigma^{*}} \text { Boot }-p\left(\frac{\alpha}{2}\right), \widetilde{\sigma^{*}}{ }_{B o o t-p}\left(1-\frac{\alpha}{2}\right)\right)
$$

respectively.

\section{Simulation Study}

In this section, we discuss the results of a simulation study comparing the performance of different estimators. Using the algorithm presented in Balakrishnan and Sandhu (1995), for a given $n, m, R_{1}, \ldots, R_{m}$, we first generate progressively Type II censored samples from the Type-II GLD. If $Y_{m: m: n}<T$ then we have Case I and the corresponding sample is $Y_{1: m: n}, \ldots, Y_{m: m: n}$. If $Y_{m: m: n}>T$, then we have Case II and we find $J$, such that $Y_{J: m: n}<T<$ $Y_{J+1: m: n}$. In this case, the censored sample is $Y_{1: m: n}, \ldots, Y_{J: m: n}$. We compute the AMLEs from (18), (19), (21) and (23). The MLEs of the parameters were then obtained by solving the nonlinear equations (7)-(10) using the function $n l m$ from the statistical software S-PLUS, in which the AMLEs were used as the starting values.

We consider different $n, m, T$ and $b$. Since $\mu$ and $\sigma$ are the location and scale parameters respectively, we have taken $\mu=0$ and $\sigma=1$ in all the cases considered. We have also used three different sampling schemes, as follows: Scheme 1: $R_{1}=\cdots=R_{m-1}=0, R_{m}=n-m$, Scheme 2: $R_{1}=n-m, R_{2}=\cdots=R_{m}=0$, Scheme 3: $R_{1}=\cdots=R_{m-1}=1$ and $R_{m}=n-2 m+1$.

In Tables 1-6, we provided the average values and variances of MLEs and AMLEs. We also provided the average confidence lengths and the corresponding $95 \%$ coverage probabilities using the asymptotic distributions of MLEs and AMLEs and bootstrap method. All the averages were computed over 1000 simulations. 
From Tables 1-6, we observe that the AMLEs and the MLEs are almost identical in terms of both biases and variances. Interestingly, in most of the cases considered, the variances of the AMLEs are smaller than the variances of the MLEs, although their biases are higher. It is observed that for fixed $n$ and $m$ as $T$ increases, the variances decrease for most of cases considered. Also, for fixed $n$ and $T$, as $m$ increases, the variances decrease.

Comparing different confidence intervals, it is observe that the coverage probabilities are smaller than the nominal level. As expected, when $m$ is small, the MLE and AMLE asymptotic confidence intervals do not work well. The asymptotic confidence intervals based on AMLEs are better the asymptotic confidence intervals based on MLEs in terms of the confidence lengths although their coverage probabilities are lower. From Tables, it is evident that the bootstrap confidence interval works quite well in most of cases considered. It provides the most coverage probabilities, especially when $n$ and $m$ are very small. It is also observed that when $m$, and $T$ increase, then the performances of different confidence intervals increase in general.

Let us now compare different estimators based on the three schemes 1 , 2 and 3. It is observed that the MLEs and AMLEs for Schemes 1 and 3 behave quite similarly in terms of biases and variances unless both $n$ and $m$ are small or $T$ is small. In most of cases considered, the variances of MLEs and AMLEs for Scheme 2 are larger than the corresponding variances for the other two schemes. We also observe that for Schemes 1 and 3, two confidence intervals based on the asymptotic distributions of the MLE and AMLE behave very similarly. These results are similar to those obtained by Kundu and Joarder (2006) in the case of the exponential distribution. 
Table 1. Means (variances) and average confidence lengths (coverage probabilities) for different sample sizes and for different schemes when $b=0.5$ and $T=1$

\begin{tabular}{|c|c|c|c|c|c|c|c|}
\hline \multirow[b]{2}{*}{$n$} & \multirow[b]{2}{*}{$m$} & \multirow[b]{2}{*}{ Scheme } & \multirow[b]{2}{*}{ Method } & \multicolumn{2}{|c|}{$\widehat{\mu}$} & \multicolumn{2}{|c|}{$\widehat{\sigma}$} \\
\hline & & & & Mean(Var) & Length(CP) & Mean(Var) & Length(CP) \\
\hline \multirow[t]{9}{*}{15} & 5 & $(0,0,0,0,10)$ & MLE & $-0.0700(0.5404)$ & $2.5421(0.753)$ & $0.9007(0.1766)$ & $1.8104(0.660)$ \\
\hline & & & AMLE & $-0.0848(0.3559)$ & $2.1987(0.706)$ & $0.8623(0.1346)$ & $1.3517(0.641)$ \\
\hline & & & Boot-p & - & $2.4134(0.805)$ & - & $1.3596(0.795)$ \\
\hline & & $(10,0,0,0,0)$ & MLE & $0.0083(2.0430)$ & $6.3274(0.853)$ & $1.0561(0.4625)$ & $3.1889(0.76)$ \\
\hline & & & AMLE & $-0.0144(1.9099)$ & $5.9218(0.827)$ & $1.0239(0.3847)$ & $2.6647(0.748)$ \\
\hline & & & Boot-p & - & $3.8136(0.910)$ & - & $1.8913(0.890)$ \\
\hline & & $(1,1,1,1,6)$ & MLE & $-0.0337(0.7288)$ & $3.1652(0.779)$ & $0.9504(0.3667)$ & $3.1404(0.687)$ \\
\hline & & & AMLE & $-0.0497(0.5312)$ & $2.7920(0.752)$ & $0.9173(0.1696)$ & $1.6006(0.674)$ \\
\hline & & & Boot-p & - & $2.4727(0.800)$ & - & $1.3942(0.765)$ \\
\hline \multirow[t]{9}{*}{25} & 10 & $(0, \cdots, 0,15)$ & MLE & $-0.0073(0.2536)$ & $2.0296(0.851)$ & $0.9864(0.2223)$ & $1.4397(0.771)$ \\
\hline & & & AMLE & $-0.0108(0.2140)$ & $1.7954(0.826)$ & $0.9479(0.1758)$ & $1.0665(0.770)$ \\
\hline & & & Boot-p & - & $1.8357(0.895)$ & - & $1.0466(0.835)$ \\
\hline & & $(15,0, \cdots, 0)$ & MLE & $0.0045(0.7488)$ & $3.7429(0.906)$ & $1.0608(0.2492)$ & $1.9728(0.820)$ \\
\hline & & & AMLE & $-0.0116(0.6954)$ & $3.5366(0.890)$ & $1.0415(0.1448)$ & $1.6090(0.821)$ \\
\hline & & & Boot-p & - & $3.5225(0.905)$ & - & $1.6434(0.910)$ \\
\hline & & $(1, \cdots, 1,6)$ & MLE & $0.0084(0.3244)$ & $2.2803(0.872)$ & $1.0086(0.1930)$ & $1.5056(0.821)$ \\
\hline & & & AMLE & $-0.0030(0.2704)$ & $2.1124(0.867)$ & $0.9925(0.0873)$ & $1.1948(0.825)$ \\
\hline & & & Boot-p & - & $1.8170(0.885)$ & - & $1.0297(0.875)$ \\
\hline \multirow[t]{18}{*}{50} & 10 & $(0, \cdots, 0,40)$ & MLE & $-0.0887(0.2289)$ & $1.8034(0.822)$ & $0.9575(0.1185)$ & $1.2319(0.764)$ \\
\hline & & & AMLE & $-0.1053(0.1883)$ & $1.6426(0.790)$ & $0.9250(0.0763)$ & $1.0459(0.762)$ \\
\hline & & & Boot-p & - & $1.5336(0.760)$ & - & $0.94280(0.795)$ \\
\hline & & $(40,0, \cdots, 0)$ & MLE & $-0.0396(1.0185)$ & $3.8355(0.883)$ & $1.0562(0.7648)$ & $1.8522(0.838)$ \\
\hline & & & AMLE & $-0.0445(0.7609)$ & $3.5529(0.885)$ & $1.0377(0.1113)$ & $1.3809(0.841)$ \\
\hline & & & Boot-p & - & $3.1549(0.900)$ & - & $1.3036(0.925)$ \\
\hline & & $(1, \cdots, 1,31)$ & MLE & $-0.0626(0.2571)$ & $1.9326(0.830)$ & $0.9869(0.1991)$ & $1.3745(0.786)$ \\
\hline & & & AMLE & $-0.0905(0.1940)$ & $1.7117(0.812)$ & $0.9513(0.0748)$ & $1.0632(0.784)$ \\
\hline & & & Boot-p & - & $1.5578(0.800)$ & - & $0.9327(0.750)$ \\
\hline & 20 & $(0, \cdots, 0,30)$ & MLE & $0.0115(0.1159)$ & $1.3346(0.895)$ & $0.9838(0.0673)$ & $0.8721(0.842)$ \\
\hline & & & AMLE & $-0.0034(0.1035)$ & $1.2379(0.875)$ & $0.9629(0.0364)$ & $0.7340(0.842)$ \\
\hline & & & Boot-p & - & $1.2549(0.890)$ & - & $0.7423(0.885)$ \\
\hline & & $(30,0, \cdots, 0)$ & MLE & $-0.0120(0.3153)$ & $2.4068(0.937)$ & $1.0635(0.1049)$ & $1.3132(0.885)$ \\
\hline & & & AMLE & $-0.0255(0.2928)$ & $2.2653(0.932)$ & $1.0399(0.0627)$ & $1.0427(0.884)$ \\
\hline & & & Boot-p & - & $1.8700(0.925)$ & - & $0.9050(0.895)$ \\
\hline & & $(1, \cdots, 1,11)$ & MLE & $-0.0001(0.1392)$ & $1.5085(0.915)$ & $1.0086(0.0654)$ & $0.9344(0.844)$ \\
\hline & & & AMLE & $-0.0284(0.1260)$ & $1.4066(0.894)$ & $0.9894(0.0399)$ & $0.7903(0.860)$ \\
\hline & & & Boot-p & - & $1.3687(0.915)$ & - & $0.7679(0.865)$ \\
\hline
\end{tabular}


Table 2. Means (variances) and average confidence lengths (coverage probabilities) for different sample sizes and for different schemes when $b=0.5$ and $T=2$

\begin{tabular}{|c|c|c|c|c|c|c|c|}
\hline \multirow[b]{2}{*}{$n$} & \multirow[b]{2}{*}{$m$} & \multirow[b]{2}{*}{ Scheme } & \multirow[b]{2}{*}{ Method } & \multicolumn{2}{|c|}{$\widehat{\mu}$} & \multicolumn{2}{|c|}{$\widehat{\sigma}$} \\
\hline & & & & Mean(Var) & Length(CP) & Mean(Var) & Length(CP) \\
\hline \multirow[t]{9}{*}{15} & 5 & $(0,0,0,0,10)$ & MLE & $-0.0643(0.4413)$ & $2.5366(0.750)$ & $0.9107(0.2323)$ & $1.6932(0.683)$ \\
\hline & & & AMLE & $-0.0933(0.3468)$ & $2.2100(0.704)$ & $0.8746(0.1296)$ & $1.3512(0.674)$ \\
\hline & & & Boot-p & - & $2.1111(0.770)$ & - & $1.2093(0.715)$ \\
\hline & & $(10,0,0,0,0)$ & MLE & $-0.0834(1.4474)$ & $5.1379(0.841)$ & $1.0276(0.4448)$ & $2.5638(0.740)$ \\
\hline & & & AMLE & $-0.0918(1.2299)$ & $4.5470(0.804)$ & $0.9906(0.2389)$ & $1.9863(0.739)$ \\
\hline & & & Boot-p & - & $3.9552(0.870)$ & - & $1.7890(0.890)$ \\
\hline & & $(1,1,1,1,6)$ & MLE & $-0.0375(0.4406)$ & $2.5558(0.752)$ & $0.9080(0.2773)$ & $1.6402(0.684)$ \\
\hline & & & AMLE & $-0.0580(0.3647)$ & $2.2613(0.729)$ & $0.8753(0.1177)$ & $1.2839(0.664)$ \\
\hline & & & Boot-p & - & $2.2966(0.800)$ & - & $1.2585(0.700)$ \\
\hline \multirow[t]{9}{*}{25} & 10 & $(0, \cdots, 0,15)$ & MLE & $-0.0204(0.2348)$ & $1.8955(0.844)$ & $0.9610(0.1776)$ & $1.2814(0.764)$ \\
\hline & & & AMLE & $-0.0351(0.1977)$ & $1.6915(0.813)$ & $0.9317(0.0674)$ & $0.9882(0.755)$ \\
\hline & & & Boot-p & - & $1.6870(0.875)$ & - & $0.9816(0.830)$ \\
\hline & & $(15,0, \cdots, 0)$ & MLE & $-0.0572(0.5509)$ & $3.1615(0.887)$ & $1.0337(0.2300)$ & $1.5981(0.827)$ \\
\hline & & & AMLE & $-0.0653(0.4886)$ & $2.9169(0.872)$ & $1.0160(0.0921)$ & $1.2548(0.822)$ \\
\hline & & & Boot-p & - & $2.7573(0.930)$ & - & $1.2230(0.940)$ \\
\hline & & $(1, \cdots, 1,6)$ & MLE & $-0.0235(0.2857)$ & $2.1897(0.883)$ & $1.0138(0.1756)$ & $1.3752(0.825)$ \\
\hline & & & AMLE & $-0.0330(0.2393)$ & $1.9480(0.857)$ & $0.9785(0.0681)$ & $1.0381(0.816)$ \\
\hline & & & Boot-p & - & $1.7668(0.885)$ & - & $0.9493(0.825)$ \\
\hline \multirow[t]{18}{*}{50} & 10 & $(0, \cdots, 0,40)$ & MLE & $-0.0772(0.2340)$ & $1.8465(0.815)$ & $0.9639(0.1430)$ & $1.3195(0.752)$ \\
\hline & & & AMLE & $-0.0970(0.1878)$ & $1.6382(0.795)$ & $0.9233(0.0761)$ & $1.0432(0.746)$ \\
\hline & & & Boot-p & - & $1.5891(0.825)$ & - & $0.9874(0.785)$ \\
\hline & & $(40,0, \cdots, 0)$ & MLE & $-0.0847(0.5365)$ & $3.1004(0.925)$ & $1.0505(0.1358)$ & $1.4124(0.863)$ \\
\hline & & & AMLE & $-0.0967(0.4774)$ & $2.8766(0.917)$ & $1.0296(0.0695)$ & $1.0903(0.845)$ \\
\hline & & & Boot-p & - & $2.8786(0.940)$ & - & $1.1506(0.960)$ \\
\hline & & $(1, \cdots, 1,31)$ & MLE & $-0.0875(0.2238)$ & $1.7955(0.808)$ & $0.9553(0.1583)$ & $1.2704(0.770)$ \\
\hline & & & AMLE & $-0.1062(0.1833)$ & $1.6177(0.784)$ & $0.9222(0.0707)$ & $1.0052(0.759)$ \\
\hline & & & Boot-p & - & $1.5602(0.810)$ & - & $0.9497(0.845)$ \\
\hline & 20 & $(0, \cdots, 0,30)$ & MLE & $-0.0151(0.1183)$ & $1.3804(0.922)$ & $1.0003(0.0634)$ & $0.8879(0.850)$ \\
\hline & & & AMLE & $-0.0295(0.1062)$ & $1.2750(0.905)$ & $0.9781(0.0369)$ & $0.7523(0.854)$ \\
\hline & & & Boot-p & - & $1.1875(0.910)$ & - & $0.6892(0.890)$ \\
\hline & & $(30,0, \cdots, 0)$ & MLE & $-0.0345(0.2677)$ & $2.1690(0.931)$ & $1.0413(0.0841)$ & $1.0775(0.889)$ \\
\hline & & & AMLE & $-0.0520(0.2452)$ & $2.0398(0.925)$ & $1.0271(0.0435)$ & $0.8557(0.888)$ \\
\hline & & & Boot-p & - & $1.8421(0.925)$ & - & $0.7976(0.945)$ \\
\hline & & $(1, \cdots, 1,11)$ & MLE & $-0.0306(0.1287)$ & $1.4230(0.917)$ & $0.9922(0.0578)$ & $0.8516(0.833)$ \\
\hline & & & AMLE & $-0.0351(0.1150)$ & $1.3128(0.909)$ & $0.9687(0.0326)$ & $0.6988(0.835)$ \\
\hline & & & Boot-p & - & $1.2796(0.900)$ & - & $0.6932(0.895)$ \\
\hline
\end{tabular}


Table 3. Means (variances) and average confidence lengths (coverage probabilities) for different sample sizes and for different schemes when $b=1$ (standard logistic case) and $T=1$

\begin{tabular}{|c|c|c|c|c|c|c|c|}
\hline \multirow[b]{2}{*}{$n$} & \multirow[b]{2}{*}{$m$} & \multirow[b]{2}{*}{ Scheme } & \multirow[b]{2}{*}{ Method } & \multicolumn{2}{|c|}{$\widehat{\mu}$} & \multicolumn{2}{|c|}{$\widehat{\sigma}$} \\
\hline & & & & Mean(Var) & Length $(\mathrm{CP})$ & Mean(Var) & Length $(\mathrm{CP})$ \\
\hline \multirow[t]{9}{*}{15} & 5 & $(0,0,0,0,10)$ & MLE & $-0.2369(0.4553)$ & $2.4175(0.677)$ & $0.8757(0.2637)$ & $1.6130(0.648)$ \\
\hline & & & AMLE & $-0.2647(0.3744)$ & $2.2308(0.668)$ & $0.8428(0.1308)$ & $1.3185(0.627)$ \\
\hline & & & Boot-p & - & $2.0526(0.615)$ & - & $1.1289(0.680)$ \\
\hline & & $(10,0,0,0,0)$ & MLE & $-0.0735(1.0803)$ & $4.4317(0.867)$ & $1.0494(0.2624)$ & $2.1318(0.820)$ \\
\hline & & & AMLE & $-0.0920(0.9901)$ & $4.2072(0.856)$ & $1.0366(0.1750)$ & $1.7852(0.795)$ \\
\hline & & & Boot-p & - & $3.5106(0.875)$ & - & $1.5619(0.870)$ \\
\hline & & $(1,1,1,1,6)$ & MLE & $-0.2040(0.4375)$ & $2.4840(0.715)$ & $0.9073(0.2046)$ & $1.5929(0.665)$ \\
\hline & & & AMLE & $-0.2267(0.3772)$ & $2.3071(0.697)$ & $0.8742(0.1209)$ & $1.3065(0.656)$ \\
\hline & & & Boot-p & - & $2.2214(0.715)$ & - & $1.1944(0.670)$ \\
\hline \multirow[t]{9}{*}{25} & 10 & $(0, \cdots, 0,15)$ & MLE & $-0.0477(0.2680)$ & $1.8464(0.818)$ & $0.9570(0.4998)$ & $1.3648(0.780)$ \\
\hline & & & AMLE & $-0.0712(0.1900)$ & $1.6738(0.803)$ & $0.9360(0.0731)$ & $1.0388(0.784)$ \\
\hline & & & Boot-p & - & $1.5717(0.825)$ & - & $0.9501(0.805)$ \\
\hline & & $(15,0, \cdots, 0)$ & MLE & $-0.0339(0.4007)$ & $2.7174(0.908)$ & $1.0559(0.2065)$ & $1.5340(0.866)$ \\
\hline & & & AMLE & $-0.0434(0.3754)$ & $2.5843(0.895)$ & $1.0384(0.0783)$ & $1.1762(0.847)$ \\
\hline & & & Boot-p & - & $2.3163(0.885)$ & - & $1.1252(0.920)$ \\
\hline & & $(1, \cdots, 1,6)$ & MLE & $-0.0681(0.2176)$ & $1.8634(0.855)$ & $0.9924(0.0939)$ & $1.1385(0.789)$ \\
\hline & & & AMLE & $-0.0860(0.1937)$ & $1.7143(0.832)$ & $0.9556(0.0643)$ & $0.9881(0.789)$ \\
\hline & & & Boot-p & - & $1.5575(0.805)$ & - & $0.9068(0.790)$ \\
\hline \multirow[t]{18}{*}{50} & 10 & $(0, \cdots, 0,40)$ & MLE & $-0.1781(0.4466)$ & $2.2017(0.774)$ & $0.9288(0.1387)$ & $1.2269(0.743)$ \\
\hline & & & AMLE & $-0.1971(0.2950)$ & $2.0252(0.768)$ & $0.9090(0.0781)$ & $1.0426(0.745)$ \\
\hline & & & Boot-p & - & $1.9180(0.825)$ & - & $0.9654(0.795)$ \\
\hline & & $(40,0, \cdots, 0)$ & MLE & $-0.0576(0.6747)$ & $2.8549(0.902)$ & $1.0761(0.5953)$ & $1.4436(0.877)$ \\
\hline & & & AMLE & $-0.0724(0.3841)$ & $2.6366(0.895)$ & $1.0554(0.0606)$ & $1.0446(0.877)$ \\
\hline & & & Boot-p & - & $2.5869(0.905)$ & - & $1.0961(0.950)$ \\
\hline & & $(1, \cdots, 1,31)$ & MLE & $-0.1622(0.3824)$ & $2.2146(0.770)$ & $0.9493(0.2105)$ & $1.2651(0.763)$ \\
\hline & & & AMLE & $-0.1802(0.2854)$ & $2.0361(0.760)$ & $0.9282(0.0755)$ & $1.0472(0.756)$ \\
\hline & & & Boot-p & - & $1.9335(0.830)$ & - & $0.9869(0.810)$ \\
\hline & 20 & $(0, \cdots, 0,30)$ & MLE & $-0.0194(0.1308)$ & $1.3355(0.879)$ & $0.9914(0.1379)$ & $0.9836(0.835)$ \\
\hline & & & AMLE & $-0.0452(0.0972)$ & $1.2102(0.865)$ & $0.9685(0.0386)$ & $0.7622(0.837)$ \\
\hline & & & Boot-p & - & $1.1015(0.825)$ & - & $0.7011(0.875)$ \\
\hline & & $(30,0, \cdots, 0)$ & MLE & $-0.0068(0.1881)$ & $1.8093(0.935)$ & $1.0496(0.0702)$ & $1.0229(0.888)$ \\
\hline & & & AMLE & $-0.0240(0.1706)$ & $1.7067(0.925)$ & $1.0336(0.0378)$ & $0.8010(0.890)$ \\
\hline & & & Boot-p & - & $1.5224(0.870)$ & - & $0.7389(0.945)$ \\
\hline & & $(1, \cdots, 1,11)$ & MLE & $-0.0247(0.1137)$ & $1.3144(0.890)$ & $0.9990(0.0736)$ & $0.8814(0.849)$ \\
\hline & & & AMLE & $-0.0447(0.0961)$ & $1.2019(0.867)$ & $0.9714(0.0329)$ & $0.7031(0.849)$ \\
\hline & & & Boot-p & - & $1.1601(0.890)$ & - & $0.6714(0.845)$ \\
\hline
\end{tabular}


Table 4. Means (variances) and average confidence lengths (coverage probabilities) for different sample sizes and for different schemes when $b=1$ (standard logistic case) and $T=2$

\begin{tabular}{|c|c|c|c|c|c|c|c|}
\hline \multirow[b]{2}{*}{$n$} & \multirow[b]{2}{*}{$m$} & \multirow[b]{2}{*}{ Scheme } & \multirow[b]{2}{*}{ Method } & \multicolumn{2}{|c|}{$\widehat{\mu}$} & \multicolumn{2}{|r|}{$\widehat{\sigma}$} \\
\hline & & & & Mean(Var) & Length(CP) & Mean(Var) & Length(CP) \\
\hline \multirow[t]{9}{*}{15} & 5 & $(0,0,0,0,10)$ & MLE & $-0.2187(0.6783)$ & $2.6420(0.721)$ & $0.8927(0.6455)$ & $1.9155(0.673)$ \\
\hline & & & AMLE & $-0.2427(0.3811)$ & $2.2701(0.690)$ & $0.8506(0.1331)$ & $1.3420(0.639)$ \\
\hline & & & Boot-p & - & $2.1113(0.755)$ & - & $1.1876(0.680)$ \\
\hline & & $(10,0,0,0,0)$ & MLE & $-0.1139(0.7767)$ & $3.6576(0.843)$ & $1.0193(0.3056)$ & $1.8564(0.793)$ \\
\hline & & & AMLE & $-0.1300(0.6243)$ & $3.2380(0.818)$ & $0.9890(0.1116)$ & $1.3609(0.777)$ \\
\hline & & & Boot-p & - & $3.2996(0.900)$ & - & $1.4469(0.905)$ \\
\hline & & $(1,1,1,1,6)$ & MLE & $-0.1927(0.4285)$ & $2.4373(0.724)$ & $0.8978(0.2240)$ & $1.5921(0.660)$ \\
\hline & & & AMLE & $-0.2122(0.3632)$ & $2.2265(0.710)$ & $0.8607(0.1165)$ & $1.2611(0.646)$ \\
\hline & & & Boot-p & - & $2.0412(0.690)$ & - & $1.1273(0.680)$ \\
\hline \multirow[t]{9}{*}{25} & 10 & $(0, \cdots, 0,15)$ & MLE & $-0.0927(0.2355)$ & $1.8435(0.807)$ & $0.9632(0.1292)$ & $1.2291(0.768)$ \\
\hline & & & AMLE & $-0.1129(0.1911)$ & $1.6837(0.793)$ & $0.9345(0.0736)$ & $1.0450(0.759)$ \\
\hline & & & Boot-p & - & $1.5833(0.890)$ & - & $0.9601(0.830)$ \\
\hline & & $(15,0, \cdots, 0)$ & MLE & $-0.0671(0.3438)$ & $2.4574(0.892)$ & $1.0415(0.1738)$ & $1.3558(0.857)$ \\
\hline & & & AMLE & $-0.0752(0.2976)$ & $2.2309(0.872)$ & $1.0099(0.0561)$ & $0.9663(0.852)$ \\
\hline & & & Boot-p & - & $2.1334(0.890)$ & - & $0.9956(0.910)$ \\
\hline & & $(1, \cdots, 1,6)$ & MLE & $-0.0559(0.2259)$ & $1.8544(0.842)$ & $0.9753(0.1306)$ & $1.2497(0.794)$ \\
\hline & & & AMLE & $-0.0788(0.1882)$ & $1.6715(0.832)$ & $0.9466(0.0626)$ & $0.9638(0.785)$ \\
\hline & & & Boot-p & - & $1.6130(0.870)$ & - & $0.9302(0.835)$ \\
\hline \multirow[t]{18}{*}{50} & 10 & $(0, \cdots, 0,40)$ & MLE & $-0.1435(0.3642)$ & $2.2490(0.786)$ & $0.9502(0.1323)$ & $1.2603(0.765)$ \\
\hline & & & AMLE & $-0.1671(0.3062)$ & $2.1023(0.781)$ & $0.9260(0.0811)$ & $1.0822(0.761)$ \\
\hline & & & Boot-p & - & $1.9410(0.765)$ & - & $0.9788(0.790)$ \\
\hline & & $(40,0, \cdots, 0)$ & MLE & $-0.0989(0.3505)$ & $2.5170(0.899)$ & $1.0549(0.0918)$ & $1.1971(0.889)$ \\
\hline & & & AMLE & $-0.1128(0.3097)$ & $2.3043(0.878)$ & $1.0300(0.0462)$ & $0.8873(0.875)$ \\
\hline & & & Boot-p & - & $2.1462(0.915)$ & - & $0.9353(0.930)$ \\
\hline & & $(1, \cdots, 1,31)$ & MLE & $-0.1801(0.3097)$ & $2.0660(0.769)$ & $0.9380(0.0987)$ & $1.1443(0.762)$ \\
\hline & & & AMLE & $-0.1896(0.2750)$ & $1.9622(0.763)$ & $0.9149(0.0727)$ & $1.0091(0.759)$ \\
\hline & & & Boot-p & - & $1.9561(0.800)$ & - & $0.9940(0.830)$ \\
\hline & 20 & $(0, \cdots, 0,30)$ & MLE & $-0.0247(0.1138)$ & $1.3255(0.882)$ & $0.9978(0.0847)$ & $0.9372(0.838)$ \\
\hline & & & AMLE & $-0.0468(0.0963)$ & $1.1983(0.868)$ & $0.9648(0.0382)$ & $0.7546(0.830)$ \\
\hline & & & Boot-p & - & $1.1592(0.885)$ & - & $0.7130(0.865)$ \\
\hline & & $(30,0, \cdots, 0)$ & MLE & $-0.0233(0.1651)$ & $1.6856(0.940)$ & $1.0388(0.0493)$ & $0.8719(0.914)$ \\
\hline & & & AMLE & $-0.0378(0.1535)$ & $1.5994(0.922)$ & $1.0247(0.0302)$ & $0.7089(0.909)$ \\
\hline & & & Boot-p & - & $1.4252(0.920)$ & - & $0.65804(0.895)$ \\
\hline & & $(1, \cdots, 1,11)$ & MLE & $-0.0433(0.1087)$ & $1.3150(0.908)$ & $1.0041(0.0628)$ & $0.8710(0.853)$ \\
\hline & & & AMLE & $-0.0622(0.0970)$ & $1.2141(0.893)$ & $0.9760(0.0331)$ & $0.7099(0.86)$ \\
\hline & & & Boot-p & - & $1.1549(0.875)$ & - & $0.6709(0.905)$ \\
\hline
\end{tabular}


Table 5. Means (variances) and average confidence lengths (coverage probabilities) for different sample sizes and for different schemes when $b=1.5$ and $T=1$

\begin{tabular}{|c|c|c|c|c|c|c|c|}
\hline \multirow[b]{2}{*}{$n$} & \multirow[b]{2}{*}{$m$} & \multirow[b]{2}{*}{ Scheme } & \multirow[b]{2}{*}{ Method } & \multicolumn{2}{|c|}{$\widehat{\mu}$} & \multicolumn{2}{|c|}{$\widehat{\sigma}$} \\
\hline & & & & Mean(Var) & Length(CP) & Mean(Var) & Length(CP) \\
\hline \multirow[t]{9}{*}{15} & 5 & $(0,0,0,0,10)$ & MLE & $-0.2792(0.7421)$ & $2.8405(0.663)$ & $0.8767(0.2170)$ & $1.5984(0.645)$ \\
\hline & & & AMLE & $-0.3156(0.4963)$ & $2.5798(0.659)$ & $0.8406(0.1362)$ & $1.3519(0.634)$ \\
\hline & & & Boot-p & - & $2.4361(0.715)$ & - & $1.2257(0.725)$ \\
\hline & & $(10,0,0,0,0)$ & MLE & $-0.1724(0.6739)$ & $3.3576(0.815)$ & $1.0099(0.1785)$ & $1.6501(0.781)$ \\
\hline & & & AMLE & $-0.1851(0.5951)$ & $3.1300(0.806)$ & $0.9851(0.1055)$ & $1.3150(0.778)$ \\
\hline & & & Boot-p & - & $3.1133(0.845)$ & - & $1.3757(0.870)$ \\
\hline & & $(1,1,1,1,6)$ & MLE & $-0.3104(0.5193)$ & $2.5934(0.669)$ & $0.8720(0.1890)$ & $1.4961(0.652)$ \\
\hline & & & AMLE & $-0.3319(0.4405)$ & $2.4237(0.653)$ & $0.8463(0.1162)$ & $1.2450(0.629)$ \\
\hline & & & Boot-p & - & $2.3146(0.710)$ & - & $1.1521(0.720)$ \\
\hline \multirow[t]{9}{*}{25} & 10 & $(0, \cdots, 0,15)$ & MLE & $-0.1005(0.3046)$ & $2.0539(0.807)$ & $0.9647(0.1963)$ & $1.3042(0.763)$ \\
\hline & & & AMLE & $-0.1275(0.2342)$ & $1.8498(0.801)$ & $0.9319(0.0747)$ & $1.0448(0.754)$ \\
\hline & & & Boot-p & - & $1.7083(0.825)$ & - & $0.9592(0.820)$ \\
\hline & & $(15,0, \cdots, 0)$ & MLE & $-0.0129(0.3182)$ & $2.3744(0.890)$ & $1.0483(0.1095)$ & $1.2959(0.870)$ \\
\hline & & & AMLE & $-0.0299(0.2952)$ & $2.2619(0.885)$ & $1.0326(0.0609)$ & $1.0256(0.870)$ \\
\hline & & & Boot-p & - & $2.0480(0.890)$ & - & $0.9943(0.910)$ \\
\hline & & $(1, \cdots, 1,6)$ & MLE & $-0.0925(0.2748)$ & $1.9227(0.823)$ & $0.9622(0.4545)$ & $1.3417(0.798)$ \\
\hline & & & AMLE & $-0.1111(0.2092)$ & $1.7427(0.812)$ & $0.9366(0.0621)$ & $0.9495(0.783)$ \\
\hline & & & Boot-p & - & $1.7073(0.845)$ & - & $0.9311(0.865)$ \\
\hline \multirow[t]{18}{*}{50} & 10 & $(0, \cdots, 0,40)$ & MLE & $-0.1770(0.5250)$ & $2.5712(0.760)$ & $0.9316(0.1064)$ & $1.1794(0.744)$ \\
\hline & & & AMLE & $-0.1920(0.4163)$ & $2.4329(0.768)$ & $0.9182(0.0815)$ & $1.0767(0.744)$ \\
\hline & & & Boot-p & - & $2.2510(0.755)$ & - & $0.9678(0.780)$ \\
\hline & & $(40,0, \cdots, 0)$ & MLE & $-0.0578(0.3136)$ & $2.3493(0.896)$ & $1.0455(0.0718)$ & $1.0978(0.897)$ \\
\hline & & & AMLE & $-0.0691(0.2954)$ & $2.2670(0.893)$ & $1.0381(0.0472)$ & $0.9041(0.884)$ \\
\hline & & & Boot-p & - & $2.0679(0.900)$ & - & $0.9346(0.915)$ \\
\hline & & $(1, \cdots, 1,31)$ & MLE & $-0.1885(0.4401)$ & $2.4864(0.758)$ & $0.9423(0.1026)$ & $1.1645(0.741)$ \\
\hline & & & AMLE & $-0.2031(0.3867)$ & $2.3648(0.753)$ & $0.9240(0.0765)$ & $1.0519(0.742)$ \\
\hline & & & Boot-p & - & $2.2323(0.825)$ & - & $0.9757(0.840)$ \\
\hline & 20 & $(0, \cdots, 0,30)$ & MLE & $-0.0719(0.1328)$ & $1.3739(0.831)$ & $0.9682(0.0522)$ & $0.8316(0.814)$ \\
\hline & & & AMLE & $-0.0871(0.1160)$ & $1.2936(0.828)$ & $0.9480(0.0380)$ & $0.7405(0.823)$ \\
\hline & & & Boot-p & - & $1.2792(0.895)$ & - & $0.7197(0.870)$ \\
\hline & & $(30,0, \cdots, 0)$ & MLE & $0.0015(0.1481)$ & $1.5970(0.911)$ & $1.0427(0.0551)$ & $0.9050(0.888)$ \\
\hline & & & AMLE & $-0.0226(0.1361)$ & $1.5137(0.924)$ & $1.0291(0.0307)$ & $0.7177(0.893)$ \\
\hline & & & Boot-p & - & $1.4277(0.890)$ & - & $0.7451(0.970)$ \\
\hline & & $(1, \cdots, 1,11)$ & MLE & $-0.0503(0.1179)$ & $1.3309(0.867)$ & $0.9866(0.0457)$ & $0.7902(0.841)$ \\
\hline & & & AMLE & $-0.0671(0.1064)$ & $1.2530(0.863)$ & $0.9618(0.0327)$ & $0.6946(0.840)$ \\
\hline & & & Boot-p & - & $1.2542(0.875)$ & - & $0.6927(0.915)$ \\
\hline
\end{tabular}


Table 6. Means (variances) and average confidence lengths (coverage probabilities) for different sample sizes and for different schemes when $b=1.5$ and $T=2$

\begin{tabular}{|c|c|c|c|c|c|c|c|}
\hline \multirow[b]{2}{*}{$n$} & \multirow[b]{2}{*}{$m$} & \multirow[b]{2}{*}{ Scheme } & \multirow[b]{2}{*}{ Method } & \multicolumn{2}{|c|}{$\widehat{\mu}$} & \multicolumn{2}{|c|}{$\widehat{\sigma}$} \\
\hline & & & & Mean(Var) & Length(CP) & Mean(Var) & Length(CP) \\
\hline \multirow[t]{9}{*}{15} & 5 & $(0,0,0,0,10)$ & MLE & $-0.2725(0.6012)$ & $2.7735(0.693)$ & $0.8833(0.2032)$ & $1.5917(0.659)$ \\
\hline & & & AMLE & $-0.3004(0.4962)$ & $2.5798(0.687)$ & $0.8494(0.1362)$ & $1.3517(0.649)$ \\
\hline & & & Boot-p & - & $2.3645(0.630)$ & - & $1.1958(0.670)$ \\
\hline & & $(10,0,0,0,0)$ & MLE & $-0.1565(0.5957)$ & $3.1799(0.823)$ & $1.0053(0.1633)$ & $1.5533(0.783)$ \\
\hline & & & AMLE & $-0.1703(0.5145)$ & $2.9493(0.824)$ & $0.9912(0.0895)$ & $1.2264(0.779)$ \\
\hline & & & Boot-p & - & $2.8204(0.870)$ & - & $1.2799(0.890)$ \\
\hline & & $(1,1,1,1,6)$ & MLE & $-0.2533(0.6266)$ & $2.7246(0.718)$ & $0.9057(0.3130)$ & $1.7126(0.683)$ \\
\hline & & & AMLE & $-0.2726(0.4584)$ & $2.5219(0.711)$ & $0.8697(0.1209)$ & $1.2955(0.659)$ \\
\hline & & & Boot-p & - & $2.3948(0.695)$ & - & $1.2188(0.695)$ \\
\hline \multirow[t]{9}{*}{25} & 10 & $(0, \cdots, 0,15)$ & MLE & $-0.0959(0.3397)$ & $2.0020(0.821)$ & $0.9536(0.1149)$ & $1.2044(0.767)$ \\
\hline & & & AMLE & $-0.1231(0.2295)$ & $1.8123(0.817)$ & $0.9245(0.0732)$ & $1.0237(0.769)$ \\
\hline & & & Boot-p & - & $1.7884(0.805)$ & - & $0.9959(0.820)$ \\
\hline & & $(15,0, \cdots, 0)$ & MLE & $-0.0610(0.2737)$ & $2.1789(0.902)$ & $1.0317(0.0937)$ & $1.1664(0.862)$ \\
\hline & & & AMLE & $-0.0751(0.2476)$ & $2.0293(0.887)$ & $1.0112(0.0485)$ & $0.8974(0.866)$ \\
\hline & & & Boot-p & - & $1.9214(0.905)$ & - & $0.9487(0.930)$ \\
\hline & & $(1, \cdots, 1,6)$ & MLE & $-0.0915(0.2443)$ & $1.8949(0.826)$ & $0.9694(0.0842)$ & $1.0954(0.784)$ \\
\hline & & & AMLE & $-0.1101(0.2140)$ & $1.7830(0.812)$ & $0.9443(0.0635)$ & $0.9715(0.785)$ \\
\hline & & & Boot-p & - & $1.6813(0.835)$ & - & $0.9314(0.810)$ \\
\hline \multirow[t]{18}{*}{50} & 10 & $(0, \cdots, 0,40)$ & MLE & $-0.2189(0.4609)$ & $2.5202(0.745)$ & $0.9282(0.0983)$ & $1.1474(0.737)$ \\
\hline & & & AMLE & $-0.2299(0.4163)$ & $2.4324(0.752)$ & $0.9138(0.0815)$ & $1.0765(0.739)$ \\
\hline & & & Boot-p & - & $2.2142(0.750)$ & - & $0.9536(0.785)$ \\
\hline & & $(40,0, \cdots, 0)$ & MLE & $-0.0797(0.2872)$ & $2.2117(0.902)$ & $1.0385(0.0681)$ & $1.0349(0.884)$ \\
\hline & & & AMLE & $-0.0963(0.2563)$ & $2.0777(0.894)$ & $1.0229(0.0397)$ & $0.8172(0.887)$ \\
\hline & & & Boot-p & - & $1.9634(0.750)$ & - & $0.8842(0.925)$ \\
\hline & & $(1, \cdots, 1,31)$ & MLE & $-0.2025(0.3973)$ & $2.3581(0.756)$ & $0.9281(0.0892)$ & $1.1017(0.747)$ \\
\hline & & & AMLE & $-0.2070(0.3740)$ & $2.2869(0.758)$ & $0.9121(0.0740)$ & $1.0173(0.737)$ \\
\hline & & & Boot-p & - & $2.1959(0.810)$ & - & $0.9620(0.810)$ \\
\hline & 20 & $(0, \cdots, 0,30)$ & MLE & $-0.0564(0.1421)$ & $1.4574(0.856)$ & $0.9940(0.0896)$ & $0.9333(0.823)$ \\
\hline & & & AMLE & $-0.0753(0.1208)$ & $1.3470(0.852)$ & $0.9661(0.0395)$ & $0.7711(0.834)$ \\
\hline & & & Boot-p & - & $1.2632(0.865)$ & - & $0.7188(0.870)$ \\
\hline & & $(30,0, \cdots, 0)$ & MLE & $-0.0012(0.1356)$ & $1.5305(0.934)$ & $1.0418(0.0450)$ & $0.8305(0.896)$ \\
\hline & & & AMLE & $-0.0147(0.1274)$ & $1.4618(0.936)$ & $1.0267(0.0269)$ & $0.6715(0.899)$ \\
\hline & & & Boot-p & - & $1.3326(0.925)$ & - & $0.6827(0.945)$ \\
\hline & & $(1, \cdots, 1,11)$ & MLE & $-0.0440(0.1248)$ & $1.3830(0.880)$ & $0.9981(0.0654)$ & $0.8777(0.843)$ \\
\hline & & & AMLE & $-0.0641(0.1087)$ & $1.2801(0.868)$ & $0.9722(0.0334)$ & $0.7097(0.849)$ \\
\hline & & & Boot-p & - & $1.2372(0.900)$ & - & $0.6860(0.885)$ \\
\hline
\end{tabular}


Table 7. Log-likelihood values for different values of $b$

\begin{tabular}{cccccc}
\hline \hline $\mathrm{b}$ & 0.5 & 1 & 1.5 & 2 & 2.5 \\
\hline $\ln L$ & -36.4300 & -35.0618 & -34.8250 & -35.0306 & -35.4229 \\
\hline
\end{tabular}

\section{Estimation of the Shape Parameter and Numer- ical Examples}

In this section, we present the analysis of two real data sets for illustrative purposes. We also discuss estimation of the shape parameter.

Example 1 (Data Set 1). The first data represent data on the time to breakdown of an insulating fluid in an accelerate test conducted at various voltages. These data are taken from Nelson (1982) [Table 6.1] and have been used earlier by Viveros and Balakrishnan (1994) and Balakrishnan and Hossain (2007). The data are as follows:

$\begin{array}{llllllll}-1.6608 & -0.2485 & -0.0409 & 0.2700 & 1.0224 & 1.1505 & 1.4231 & 1.5411 \\ 1.5789 & 1.8718 & 1.9947 & 2.0806 & 2.1126 & 2.4898 & 3.4578 & 3.4818 \\ 3.5237 & 3.6030 & 4.2889 & & & & & \end{array}$

For a given $b$, let us first fit the Type-II GLD to above data set. As mentioned by Tiku and Akkaya (2004), we can identify a plausible value of the shape parameter $b$ by using log-likelihood values, Q-Q plots, goodness of fit tests, or by matching (approximately) the sample skewness and kurtosis with the corresponding value of the distribution. In Table 7 , we have computed the log-likelihood values for a series of values of $b$ based on above data set. The value $\widehat{b}$ which maximizes $\ln L$ is the required estimate. From Table 7, we can choose $b=1.5$ as a plausible value of the shape parameter.

Another method to identify a plausible value of the shape parameter $b$ is to use the profile likelihood function to get its estimate. When the parameter $b$ is unknown, the maximum profile likelihood estimate $\widehat{b}_{P}$ may be obtained by maximizing the log-likelihood $\ell_{p}(b)=l(\widetilde{\mu}(b), \widetilde{\sigma}(b), b)$ with respect to $b$. Figure 1 provides the plot of the profile likelihood function with respect to $b$ for the above data. From Figure 1, it is observed that the maximum profile likelihood estimate of $b$ should be close to $b=1.5$.

Now, we check the validity of the Type-II GLD with $b=1.5$ based on the Kolmogorov-Smirnov (K-S) test. It is observed that the K-S distance and 
the corresponding p-value are respectively

$$
K-S=0.1399, \quad \text { and } \quad p-\text { value }=0.8281 .
$$

So, the fit of the Type-II GLD with $b=1.5$ to the above data set is quite reasonable.

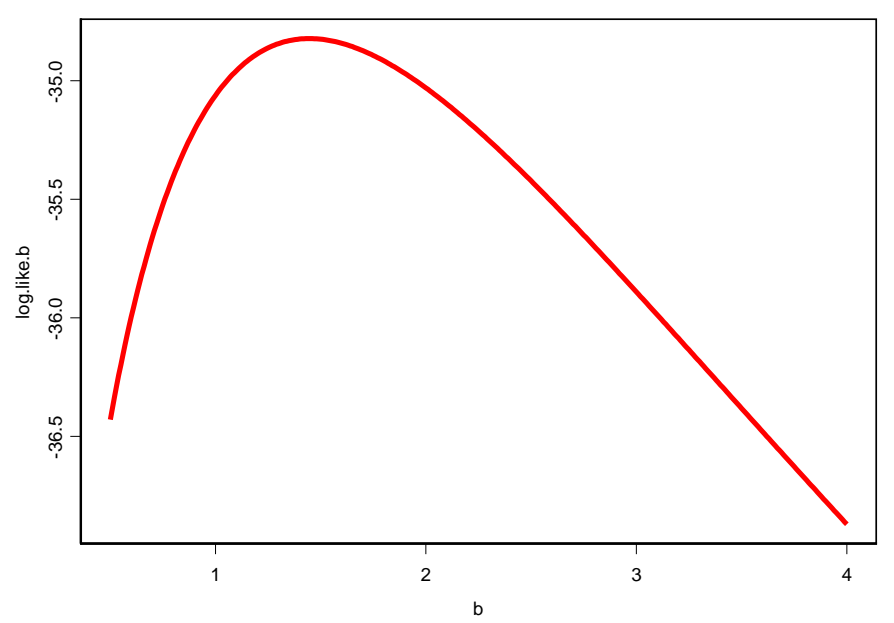

Figure 1. Plot of $\ell_{p}(b)$.

Here we have $n=19$ and $b=1.5$. Now, for the given data set, we consider two cases as follows:

Case I : $m=11, T=2,\left(R_{1}, \ldots, R_{m}\right)=(0,0, \ldots, 0,8)$. In this case, the Type-II progressively hybrid censored sample is:

$\begin{array}{llllllll}-1.6608 & -0.2485 & -0.0409 & 0.02700 & 1.0224 & 1.1505 & 1.4231 & 1.5411\end{array}$ $1.5789 \quad 1.8718 \quad 1.9947$.

As you see $y_{11: 11: 19}<T$, therefore we have the Case I of our study. Then

$$
\text { AMLE : } \quad \widetilde{\mu}=2.2692, \quad \widetilde{\sigma}=0.9151, \quad V^{*}=\left(\begin{array}{cc}
0.1509 & 0.0433 \\
0.0433 & 0.0549
\end{array}\right),
$$


Table 8. Location and scale parameters, K-S, and $p$ values of the fitted standard logistic distribution for Data Set 2

\begin{tabular}{cccc}
\hline \hline Location Parameter & Scale Parameter & K-S & P-Value \\
\hline 10.010 & 0.843 & 0.2155 & 0.4401 \\
\hline
\end{tabular}

$$
M L E: \quad \widehat{\mu}=2.2717, \quad \widehat{\sigma}=0.9033, \quad V=\left(\begin{array}{ll}
0.1463 & 0.0422 \\
0.0422 & 0.0579
\end{array}\right) \text {. }
$$

The corresponding \%95 confidence intervals based on asymptotic distributions of AMLEs and MLEs and Bootstrap confidence intervals for $\mu$ and $\sigma$ are $(1.5077,3.0306),(0.4557,1.3746),(1.5220,3.0215),(0.4316,1.3750)$, $(1.3809,3.2880)$ and $(0.4172,1.6206)$ respectively.

Case II: Now consider that $m=11, T=1.5$ and the $R_{i}$ 's are same as before. In this case, the progressively hybrid censored sample is:

$\begin{array}{lllllll}-1.6608 & -0.2485 & -0.0409 & 0.02700 & 1.0224 & 1.1505 & 1.4231 .\end{array}$

Since $y_{11: 11: 19}>T$, therefore we have the Case II of our study. From the above sample data, we obtain $J=7$ and $R_{J}^{*}=12$. Then

$$
\begin{array}{ccc}
A M L E: & \widetilde{\mu}=2.5697, \quad \widetilde{\sigma}=1.0611, \quad V^{*}=\left(\begin{array}{ll}
0.4150 & 0.1640 \\
0.1640 & 0.1268
\end{array}\right), \\
M L E: & \widehat{\mu}=2.5675, \quad \widehat{\sigma}=1.0510, \quad V=\left(\begin{array}{ll}
0.4126 & 0.1644 \\
0.1644 & 0.1316
\end{array}\right) .
\end{array}
$$

The corresponding \%95 confidence intervals based on asymptotic distributions of AMLEs and MLEs and Bootstrap confidence intervals for $\mu$ and $\sigma$ are $(1.3069,3.8325),(0.3631,1.7592),(1.3084,3.8265),(0.3399,1.7621)$, $(1.5786,4.5590)$ and $(0.3695,1.9184)$ respectively.

Example 2 (Data Set 2). The following 15 observations, from Tiku and Akkaya (2004), represent the measurements of a certain characteristic in blood cells. 


$\begin{array}{llllllll}8.921 & 8.982 & 9.048 & 9.262 & 9.689 & 9.715 & 9.774 & 9.830 \\ 10.128 & 10.485 & 10.591 & 10.766 & 10.840 & 10.881 & 11.263 . & \end{array}$

We fitted the standard logistic distribution to the above data set. We presented the estimated location and scale parameters, Kolmogorov-Smirnov (K-S) distances between the fitted and the empirical distribution functions, and corresponding $p$ value in Table 8 . From Table 8 , it is clear that the standard logistic distribution (Type-II GLD with $b=1$ ) fits quite well to the data.

Case I: In this case $n=15, m=5, T=11,\left(R_{1}, \ldots, R_{m}\right)=(3,1,2,1,3)$. Thus, the Type-II progressively hybrid censored sample is:
8.921
9.689
9.774
10.485
10.766

As you see $y_{5: 5: 15}<T$ therefore we have the Case I of our study. So, we obtain

$$
\begin{array}{ccc}
\text { AMLE }: & \widetilde{\mu}=10.6809, \quad \tilde{\sigma}=0.5271, \quad V^{*}=\left(\begin{array}{ll}
0.10899 & 0.02703 \\
0.02703 & 0.03035
\end{array}\right), \\
M L E: & \widehat{\mu}=10.6912, \quad \widehat{\sigma}=0.5168, \quad V=\left(\begin{array}{ll}
0.10959 & 0.02431 \\
0.02431 & 0.03539
\end{array}\right) .
\end{array}
$$

The corresponding $\% 95$ confidence intervals based on asymptotic distribution of AMLEs, MLEs and bootstrap confidence intervals for $\mu$ and $\sigma$ are $(10.0339,11.3281),(0.1856,0.8685),(10.0463,11.3360),(0.1622,0.8714)$, $(9.9237,11.2541)$ and $(0.1725,0.9293)$ respectively .

Case II: In this case $n=15, m=5, T=10,\left(R_{1}, \ldots, R_{m}\right)=(3,1,2,1,3)$. Thus, the Type-II progressively hybrid censored sample is:
8.921
9.689
9.774 .

As you see $y_{5: 5: 15}>T$ therefore we have the Case II of our study. From the above sample data, we obtain $J=3$ and $R_{J}^{*}=6$. Hence, we have

$$
\text { AMLE : } \quad \widetilde{\mu}=10.3735, \quad \widetilde{\sigma}=0.4097, \quad V^{*}=\left(\begin{array}{ll}
0.07582 & 0.00988 \\
0.00988 & 0.00948
\end{array}\right),
$$




$$
M L E: \quad \widehat{\mu}=10.3654, \quad \widehat{\sigma}=0.3925, \quad V=\left(\begin{array}{ll}
0.00331 & 0.00013 \\
0.00013 & 0.00029
\end{array}\right) .
$$

The corresponding \%95 confidence intervals based on asymptotic distribution of AMLEs, MLEs and bootstrap confidence intervals for $\mu$ and $\sigma$ are $(9.8338,10.9133), \quad(0.2188,0.6006), \quad(9.6367,11.0941), \quad(0.0046,0.7805)$, $(9.8484,11.3185)$ and $(0.1132,0.7392)$ respectively .

\section{Conclusions}

In this paper, we have considered point and interval estimation for the location and scale parameters of the Type-II GLD based on progressively TypeII hybrid censored data. The maximum likelihood method yields equations that do not provide explicit solutions for the parameters. We proposed the approximate MLEs of the unknown parameters which can be obtained in explicit forms. The results of the Monte Carlo simulation study show that the approximate estimators and the MLEs are almost identical in terms of bias and variance.

Although we have considered approximate solutions for MLEs when the lifetime distribution is Type-II GLD, but the methods can be extended to other generalized logistic distributions as well. In addition, the methods presented in this work could be extended to progressively Type-I hybrid censored data with some straightforward modifications. Note that also that all of the results obtained in this study can be specialized to the hybrid censored data (for $R_{i}=0, i=1,2, \ldots, m(J)$ ).

\section{Acknowledgement}

The authors would like to thank the referees for their helpful comments which improved the paper.

\section{References}

Asgharzadeh, A. (2006). Point and Interval Estimation for a Generalized Logistic Distribution under Progressive Type-II Censoring. Communications in Statistics-Theory and Methods $\mathbf{3 5}$, 1685-1702.

Asgharzadeh, A., Kazemi, M. and Kundu, D. (2017). Estimation of $P(X>Y)$ for Weibull Distribution based on Hybrid Censored Samples. International Journal of System Assurance Engineering and Management 8, 489-498. DOI: 10.1007/s13198-015-0390-2. 
Asgharzadeh, A., Valiollahi, R. and Kundu, D. (2015). Prediction for Future Failures in Weibull Distribution under Hybrid Censoring. Journal of Statistical Computation and Simulation, 85, 824-838.

Balakrishnan, N. and Aggarwala, R. (2000). Progressive Censoring: Theory, Methods and Applications; Boston: Birkhauser.

Balakrishnan, N. and Hossain, A. (2007). Inference for the Type-II Generalized Logistic Distribution under Progressive Type-II Censoring. Journal of Statistical Computation and Simulation 77 , 1013-1031.

Balakrishnan, N. and Kannan, N. (2000). Point and Interval Estimation for the Parameters of the Logistic Distribution based on Progressively Type-II Censored Samples. In Handbook of Statistics-Vol. 20 (Eds., N. Balakrishnan and C. R. Rao), 431-456.

Balakrishnan, N. and Leung, M.Y. (1988). Order Statistics from the Type-I Generalized Logistic Distribution. Communications in Statistics-Simulation and Computatio, 17, 25-50.

Balakrishnan, N. and Sandhu, R.A. (1995). A Simple Simulational Algorithm for Generating Progressive Type-II Censored Samples. Amer. Statist., 49, 229-230.

Bayat Mokhtari, E., Habibi Rad, A. and Yousefzadeh, F. (2011). Inference for Weibull Distribution based on Progressively Type-II Hybrid Censored Data. Journal of Statistical Planning and Inference, 141, 2824-2838

Chen, S. and Bhattachacharya, G.K. (1988). Exact Confidence Bounds for an Exponential Parameter under Hybrid Censoring. Communications in Statistics-Theory and Methods, 17, 1857-1870.

Draper, N. and Guttman, I. (1987). Bayesian Analysis of Hybrid Life Tests with Exponential Failure Times. Annals of the Institute of Statistical Mathematics, 39, 219-225.

Ferguson, T.S. (1996). A Course in Large Sample Theory; London: Chapman and Hall.

Efron, B. (1982). The Jacknife, the Bootstrap and Other Resampling Plans; CBMSNSF Regional Confrence Series in Applied Mathematics, 38, SIAM, Philadelphia, PA.

Efron, B. (1979). Bootstrap Methods, Another Look at the Jackknife. The Annals of Statistics, 7, 1-26.

Epstein, B. (1954). Truncated Life Tests in the Exponentioal Case. Annals os Statistics, 25, $555-564$.

Fairbanks, K., Madson, R. and Dykstra, R. (1982). A Confidence Interval for an Exponential Parameter from a Hybrid Life Test. Journal of the American Statistical Association, 77, $137-140$. 
Gurunlu Alma, O. and Arabi Belaghi, R. (2016). On the Estimation of the Extreme Value and Normal Distribution Parameters based on Progressive Type-II Hybrid-censored Data. Journal of Statistical Computation and Simulation, 86, 569-596.

Gupta, R.D. and Kundu, D. (1998). Hybrid Censoring Schemes with Exponential Failure Distribution. Communications in Statistics-Theory and Methods 27, 3065-3083.

Hemmati, F. and Khorram, E. (2013). Statistical Analysis of the Log-normal Distribution under Type-II Progressive Hybrid Censoring Schemes. Communications in Statistics-Theory and Methods 42, 52-75.

Joarder, A., Krishna, H. and Kundu, D. (2009). On Type-II Progressively Hybrid Censoring. Journal of Modern Applied Statistical Methods, 8(2), 534-546.

Kayal, T., Tripathi, Y.M., Rastogi, M.K. and Asgharzadeh, A. (2017). Inference for Burr XII Distribution under Type-I Progressive Hybrid Censoring. Communications in StatisticsSimulation and Computation, 46, 7447-7465, DOI: /10.1080/03610918.2016.1241405.

Kundu, D. and Joarder, A. (2006) Analysis of Type-II Progressively Hybrid Censored Data. Computational Statistics and Data Analysis, 50, 2509-2528.

Kundu D. (2007). On Hybrid Censored Weibull Distribution. Journal of Statistical Planning and Inference, 137, 2127-2142.

Lin, C.T., Ng, H.K.T. and Chan, P.S. (2009). Statistical Inference of Type-II Progressively Hybrid Censored Data with Weibull Lifetimes. Communications in Statistics - Theory and Methods, 38, 1710-1729.

Nelson, W. (1982). Applied Life Data Analysis; New York: John Wiley and Sons.

Tiku, M.L. and Akkaya, A.D. (2004). Robust Estimation and Hypothesis Testing, New Age International Limited Publishers. New Delhi.

Valiollahi, R., Asgharzadeh, A. and Kundu, D. (2017). Prediction of Future Failures for Generalized Exponential Distribution under Type-I or Type-II Hybrid Censoring. Brazilian Journal of Probability and Statistics. 31, 41-61.

Viveros, R. and Balakrishnan, N. (1994). Interval Estimation of Parameters of Life Progressively Censored Data. Technometrics, 36, 84-91.

\section{Appendix A}

To prove $\beta_{i}>0$, it suffices to show that $h_{1}^{\prime}(x)<0$. The function $h_{1}(x)$ may be written as

$$
h_{1}(x)=\frac{\partial}{\partial x} \ln f(x)=\frac{-b+e^{-x}}{e^{-x}+1} .
$$


Therefore, we have

$$
h_{1}^{\prime}(x)=\frac{-(b+1) e^{-x}}{\left(1+e^{-x}\right)^{2}}<0 .
$$

In order to show that $\gamma_{i}>0$ and $\gamma_{d}^{*}>0$, the function $h_{2}(x)$ may be written as

$$
h_{2}(x)=-\frac{\partial}{\partial x} \ln (1-F(x))=\frac{b}{1+e^{-x}} .
$$

So

$$
h_{2}^{\prime}(x)=\frac{b e^{-x}}{\left(1+e^{-x}\right)^{2}}>0 .
$$

Consequently, we have $\gamma_{i}>0$ and $\gamma_{d}^{*}>0$.

\section{Mina Azizpour}

Department of Statistics,

University of Mazandaran,

Babolsar, Iran.

email:minaazizpoor@gmail.com

\section{A. Asgharzadeh}

Department of Statistics,

University of Mazandaran,

Babolsar, Iran.

email: a.asgharzadeh@umz.ac.ir 
